\title{
APLICACIÓN DE 1-METILCICLOPROPENO (1-MCP) Y SU EFECTO EN CIRUELA MEXICANA (Spondias purpurea L.)
}

\author{
APPLICATION OF 1-METHYLCYCLOPROPENE (1-MCP) ON MEXICAN \\ PLUM (Spondias purpurea L.)
}

\author{
Jorge A. Osuna García ${ }^{\star 1}$, Ma. Hilda Pérez Barraza ${ }^{1}$, Víctor Vázquez Valdivia ${ }^{\dagger}$ y Rafael Gómez Jaimez ${ }^{1}$
}

${ }^{1}$ C.E. Santiago Ixcuintla, Instituto Nacional de Investigaciones Forestales. Km 6 Entronque Carretera Internacional a Santiago Ixcuintla. Apdo. Postal No. 100. 63300, Santiago Ixcuintla, Nayarit, México. Tel y Fax 01 (323) 2350710.

*Autor para correspondencia (osuna.jorgealberto@inifap.gob.mx, josunaga@hotmail.com)

\section{RESUMEN}

La ciruela amarilla mexicana (Spondias purpurea L.) es un fruto altamente perecedero que se comercializa principalmente en fresco en mercados cercanos a su zona de producción. Debido a su corta vida de anaquel (1 a $3 \mathrm{~d}$ al ambiente) y al nulo uso de refrigeración por falta de infraestructura, se pierde hasta $40 \%$ de la producción. Aquí se evaluó el efecto del 1-Metilciclopropeno (1-MCP) sobre la vida de anaquel y calidad de frutos de ciruela mexicana fenotipo amarillo almacenados en condiciones de simulación de mercadeo. Se recolectaron frutos en dos estados de madurez (sazón y tres cuartos) en un huerto ubicado en El Venado, Mpio. de Ruiz, Nayarit, México. Los frutos fueron lavados con agua corriente, secados al ambiente y seleccionados por sanidad, uniformidad de tamaño y color; se eliminaron los frutos deformes, con daños mecánicos o de plagas y enfermedades. El 1-MCP se aplicó en dosis de 100, 200 ó $300 \mathrm{~nL} \mathrm{~L}^{-1}$ por $12 \mathrm{~h}$ a temperatura ambiente, en cámaras experimentales herméticas de $0.512 \mathrm{~m}^{3}$, más un testigo sin 1-MCP; los frutos se almacenaron luego bajo simulación de mercadeo $\left(22 \pm 2{ }^{\circ} \mathrm{C}\right.$; $70 \pm 10 \% \mathrm{HR}$ ) hasta por $9 \mathrm{~d}$. Las variables medidas en los frutos fueron: velocidad de respiración, pérdida de peso, color externo, firmeza y sólidos solubles totales. El 1-MCP en dosis de 100, 200 ó $300 \mathrm{~nL} \mathrm{~L}^{-1}$ alargó la vida útil de los frutos de ciruela mexicana fenotipo amarillo hasta por $3 \mathrm{~d}$ adicionales con respecto al testigo, para alcanzar así 9 y $7 \mathrm{~d}$ de vida de anaquel en frutos sazones y tres cuartos, respectivamente. El 1-MCP disminuyó la respiración y la pérdida de peso de frutos sazones, pero no de frutos en tres cuartos de maduración, retrasó el desarrollo del color externo y mantuvo firmeza del fruto, sin afectar su contenido de sólidos solubles totales.

Palabras clave: Spondias purpurea, 1-MCP, calidad, estado de madurez, firmeza.

\section{SUMMARY}

Mexican yellow plum (Spondias purpurea L.) is a highly perishable fruit which is mainly sold mostly as a fresh fruit in regional markets. Because of its short shelf life of only 1 to $3 \mathrm{~d}$ at ambient temperature and the lack of refrigeration for postharvest store, ca. $40 \%$ of the production is lost. In this study the effect of 1-Methylcyclopropene (1-MCP) on shelf life and quality of Mexican plum yellow phenotype stored under market simulation was evaluated. Ripe and 3/4 ripe fruits were collected from a commercial orchard located in El Venado, Ruiz County, Nayarit State, México. Fruits were washed with tap water, dried, and selected for healthy and uniformity according to size and color, removing unhealthy and damaged fruits. 1-MCP was applied at 100, 200 or $300 \mathrm{~nL} \mathrm{~L}^{-1}$ for $12 \mathrm{~h}$ at ambient temperature inside sealed experimental chambers of $0.512 \mathrm{~m}^{3}$, keeping an untreated control. After the 1-MCP application, fruits were stored simulating market conditions $\left(22 \pm 2{ }^{\circ} \mathrm{C}\right.$;
$70 \pm 10 \% \mathrm{RH})$ up to $9 \mathrm{~d}$. The variables measured in the fruits were: respiration rate, weight loss, external color, firmness and total soluble solids. 1-MCP at rates of 100, 200 or $300 \mathrm{~nL} \mathrm{~L}^{-1}$ were able to extend shelf life and to maintain the quality of Mexican plum yellow phenotype fruits up to 3 additional days compared to the control, thus achieving 9 and 7 $\mathrm{d}$ of shelf life for ripe and $3 / 4$ ripe fruits, respectively. 1-MCP decreased the respiration rate and the weight loss of ripe fruits, but not in $3 / 4$ ripe fruits. 1-MCP delayed also the development of external color and maintained fruit firmness, without affecting its total soluble solids content.

Index words: Spondias purpurea, 1-MCP, quality, ripening stage, firmness.

\section{INTRODUCCIÓN}

La ciruela amarilla mexicana (Spondias purpurea L.) es nativa de México, donde se reportan 15626 ha cultivadas con un volumen de producción de 76052 t y un valor de 216.6 millones de pesos; en el Estado de Nayarit se cultivan 747 ha, con un volumen de $3613 \mathrm{t}$ y un valor de 12.9 millones de pesos (SIP-SAGARPA, 2007). El fruto de la ciruela es altamente perecedero, y se comercializa principalmente en fresco en mercados cercanos a su zona de producción. Debido a su corta vida de anaquel, entre 1 y $3 \mathrm{~d}$ al ambiente $(\approx$ $30{ }^{\circ} \mathrm{C} ; 70$ a $80 \% \mathrm{HR}$ ) y a la nula utilización de refrigeración por falta de infraestructura, se pierde hasta $40 \%$ de la producción; dicha pérdida representa, tan sólo para Nayarit, más de 5 millones de pesos anuales (SAGARPA, 2008).

Una alternativa para alargar vida de anaquel es cosechar en estado de madurez adecuado (Pérez-López et al., 2004) y almacenar a bajas temperaturas en recipientes herméticos (Montalvo-González et al., 2011). Otra alternativa es el uso del 1-MCP, un gas inocuo (EPA, 2002) que bloquea la acción del etileno e impide que se desencadenen las reacciones que conllevan al proceso de maduración, tales como: disminución de la firmeza en los tejidos, desintegración de la pared celular, degradación de pigmentos y desdoblamiento de almidones a azúcares solubles (Sisler y Serek, 
1997 y 1999).

El descubrimiento y comercialización del 1-MCP como inhibidor del etileno fue desarrollado en la Universidad Estatal de Carolina del Norte, EE. UU. (Sisler y Blankenship, 1996). A partir de entonces ese compuesto se ha experimentado con buenos resultados en una gran variedad de frutos tropicales y subtropicales, tales como aguacate Persea americana L. (Feng et al., 2000; Kluge et al., 2002; Osuna-García et al., 2005a), mango Mangifera indica L. (Jiang y Joyce, 2000; Hofman et al., 2001; Osuna-García et al., 2005b), papaya Carica papaya L. (Hofman et al., 2001; Osuna-García et al., 2005c), chicozapote Manilkara sapota (L.) P. Royen (Quiping et al., 2006; Arévalo-Galarza et al., 2007) y jaca Artocarpus heterophyllus Lam (Mata-Montes de Oca et al., 2007).

En ciruela de clima templado tipo europea (Prunus domestica L.) y tipo japonesa (Prunus salicilina L), el 1-MCP disminuye la velocidad de respiración, la producción de etileno, el ablandamiento y el cambio de color de cáscara y pulpa, sin afectar la pérdida de peso, la acidez ni el contenido de sólidos solubles totales (Dong et al., 2002; Argenta et al., 2003; Martínez-Romero et al., 2003; Salvador et al., 2003; Valero et al., 2004; Menniti et al., 2004; Manganaris et al., 2007; Khan y Singh, 2008). Por su parte, Nava y Ramírez (2008; Com. pers. $^{1}$ ) sometieron dos fenotipos de ciruela mexicana (roja y amarilla) al 1-MCP y reportaron que la aplicación de éste no prolongó la vida de anaquel del fruto en condiciones de almacenamiento al ambiente $\left(21 \pm 1^{\circ} \mathrm{C}\right)$.

Con base en lo anterior, el presente trabajo se hizo con el objetivo de evaluar el efecto del 1-Metilciclopropeno (1MCP) en la vida de anaquel y calidad de la ciruela mexicana fenotipo amarillo, almacenada en condiciones simuladas de mercadeo.

\section{MATERIALES Y MÉTODOS}

En junio de 2007 se recolectaron frutos de ciruela fenotipo amarillo en dos estados de madurez: sazón (frutos con epidermis verde y un ligero amarillamiento de las venas) y tres cuartos (frutos con al menos $75 \%$ de epidermis amarilla), de un huerto comercial ubicado en la población de El Venado, Municipio de Ruiz, Nayarit, México. Los frutos colectados fueron trasladados el mismo día de cosecha al laboratorio de postcosecha ubicado en Santiago Ixcuintla, Nayarit. Los frutos fueron lavados con agua corriente, secados al ambiente y seleccionados por sanidad, uniformidad

\footnotetext{
${ }^{1}$ Nava Z A, Ramírez R J V (2008) Respuesta del fruto de ciruela mexicana (Spondias purpurea L.) a tratamientos con 1-Metilciclopropeno (1-MCP) en postcosecha. Tesis de Licenciatura. Universidad Autónoma Chapingo. Dpto. de Ing. Agroindustrial. 71 p.
}

de tamaño y color. Se eliminaron los frutos deformes, con daños mecánicos y los que presentaban señales visibles de plagas o enfermedades.

Los frutos así seleccionados fueron inmediatamente sometidos a la aplicación de 100, 200 ó $300 \mathrm{~nL} \mathrm{~L}^{-1}$ de 1-MCP por $12 \mathrm{~h}$ a temperatura ambiente, en cámaras plásticas experimentales herméticas de $0.512 \mathrm{~m}^{3}$, más un tratamiento testigo sin aplicar. Para la aplicación del 1-MCP se utilizaron tabletas que liberan $100 \mathrm{~nL} \mathrm{~L}^{-1}$ de ingrediente activo. Para lograr las dosis antes indicadas, en cada cámara en forma independiente y simultánea se utilizaron una, dos o tres tabletas disueltas en $18 \mathrm{~mL}$ de solución activadora compuesta de ácido cítrico (8\%), citrato de sodio (2\%) y agua (90\%), más una tableta efervescente compuesta de bicarbonato de sodio (95\%), polietilenglicol (3\%) e hidroxipropil celulosa éter (2\%), para así liberar 100, 200 ó 300 $n L^{-1}$, respectivamente (Rohm y Haas, 2002). Después de la aplicación del 1-MCP, los frutos se almacenaron en condiciones simuladas de mercadeo $\left(22 \pm 2{ }^{\circ} \mathrm{C} ; 70 \pm 10 \% \mathrm{HR}\right)$ hasta por $9 \mathrm{~d}$.

Las variables analizadas fueron: velocidad de respiración, pérdida de peso, color externo, firmeza y sólidos solubles totales. La velocidad de respiración se determinó en un sistema estático con un detector de $\mathrm{CO}_{2}$ (Q-TRAK PLUS ${ }$ modelo 8552; TSI Inc. MN, USA), en el que se colocó un fruto de peso conocido dentro de un frasco de volumen conocido y cerrado herméticamente; este procedimiento se hizo por triplicado. Después de $30 \mathrm{~min}$ se leyó la cantidad de $\mathrm{CO}_{2}$ acumulada y se le restó el valor inicial; los resultados se expresaron en mg de $\mathrm{CO}_{2} \mathrm{~kg}^{-1} \mathrm{~h}^{-1}$. La pérdida de peso acumulada se midió con una balanza digital (VI4800®; ACCULAB, USA) con sensibilidad de 0.1 g. Para ello, 20 frutos marcados se pesaron diariamente durante toda la etapa de evaluación. La diferencia en peso y su relación con el peso inicial se expresó como pérdida de peso, en porcentaje.

El color de la epidermis se determinó en la parte ecuatorial de los frutos con un colorímetro portátil (Color Reader CR 10®; Konica Minolta Sensing Inc., Japan), y se reportó como ángulo de matiz. La firmeza de la pulpa se midió con un penetrómetro (Chatillón DFE-050®; Ametek Instruments, Largo, FL, USA) adaptado con punzón cilíndrico de $6 \mathrm{~mm}$ de diámetro; la lectura se hizo en la parte ecuatorial del fruto después de eliminar $0.5 \mathrm{~cm}$ de la cáscara, y los datos se expresaron en Newtons (N). Los sólidos solubles totales (SST) se determinaron directamente en tres a cuatro gotas de jugo por fruto, con un refractómetro digital (Atago PAL-1®; Atago Inc. Bellevua, WA, USA) con corrección de temperatura; las mediciones se expresaron en ${ }^{\circ} \mathrm{Bx}$ (AOAC, 1984). 
Los análisis de varianza se efectuaron considerando el efecto de tratamientos dentro de grado de madurez (Dowdy y Wearden, 1991). Las medias y el error estándar dentro de cada muestreo se obtuvieron con Proc Means del modelo lineal general (GLM) del SAS (Rebolledo, 2002). Para respiración se consideraron tres repeticiones con un fruto como unidad experimental, en tanto que para color externo, firmeza y sólidos solubles totales se utilizaron seis repeticiones. En la variable pérdida de peso se consideraron 20 frutos por tratamiento. Los datos se graficaron con el programa Sigma Plot ${ }^{\circledR}$ for Windows V. 2.01.

\section{RESULTADOS Y DISCUSIÓN}

\section{Velocidad de respiración}

La velocidad de respiración de frutos de ciruela mexicana fenotipo amarillo almacenados bajo simulación de mercadeo $\left(22 \pm 2{ }^{\circ} \mathrm{C} ; 75 \pm 10 \% \mathrm{HR}\right)$ en respuesta a los tratamientos, se muestra en la Figura 1. En los frutos testigo cosechados en estado de madurez sazón se observó una producción inicial de $\mathrm{CO}_{2}$ de $50.8 \mathrm{mg} \mathrm{kg}^{-1} \mathrm{~h}^{-1}$ que se incrementó a 103.3 mg de $\mathrm{CO}_{2} \mathrm{~kg}^{-1} \mathrm{~h}^{-1}$ en el día tres de almacenamiento, para posteriormente disminuir a valores entre 35 y $40 \mathrm{mg}$ de $\mathrm{CO}_{2}$ $\mathrm{kg}^{-1} \mathrm{~h}^{-1}$ hasta el término de su almacenamiento. Lo anterior denota un comportamiento climatérico de este fenotipo de ciruela, que coincide con el citado por Montalvo-González et al. (2011) para este mismo tipo de ciruela y con lo reportado por Canto-Pereira et al. (2000) para ciruela mexicana (Spondia purpurea L.) y ciruela amarilla (S. mombin L.). Sin embargo, contrasta con el referido por Pérez-López et al. (2004) quienes consideraron que la ciruela mexicana ( $S$. purpurea L.) es un fruto no climatérico porque en ninguno de los tres estados de maduración observaron cambios significativos en la velocidad de respiración durante el periodo de almacenamiento.

En cuanto a los frutos tratados, fue notorio que cualquiera de las dosis del 1-MCP suprimió la aparición del pico climatérico de respiración, puesto que los valores de $\mathrm{CO}_{2}$ oscilaron desde 45 hasta $65 \mathrm{mg} \mathrm{kg}^{-1} \mathrm{~h}^{-1}$ durante el almacenamiento. En cambio, Nava y Ramírez (2008) no detectaron efecto del 1-MCP para esta variable en frutos de ciruela mexicana de los fenotipos rojo y amarillo. Sin embargo, en frutos tropicales climatéricos altamente perecederos como
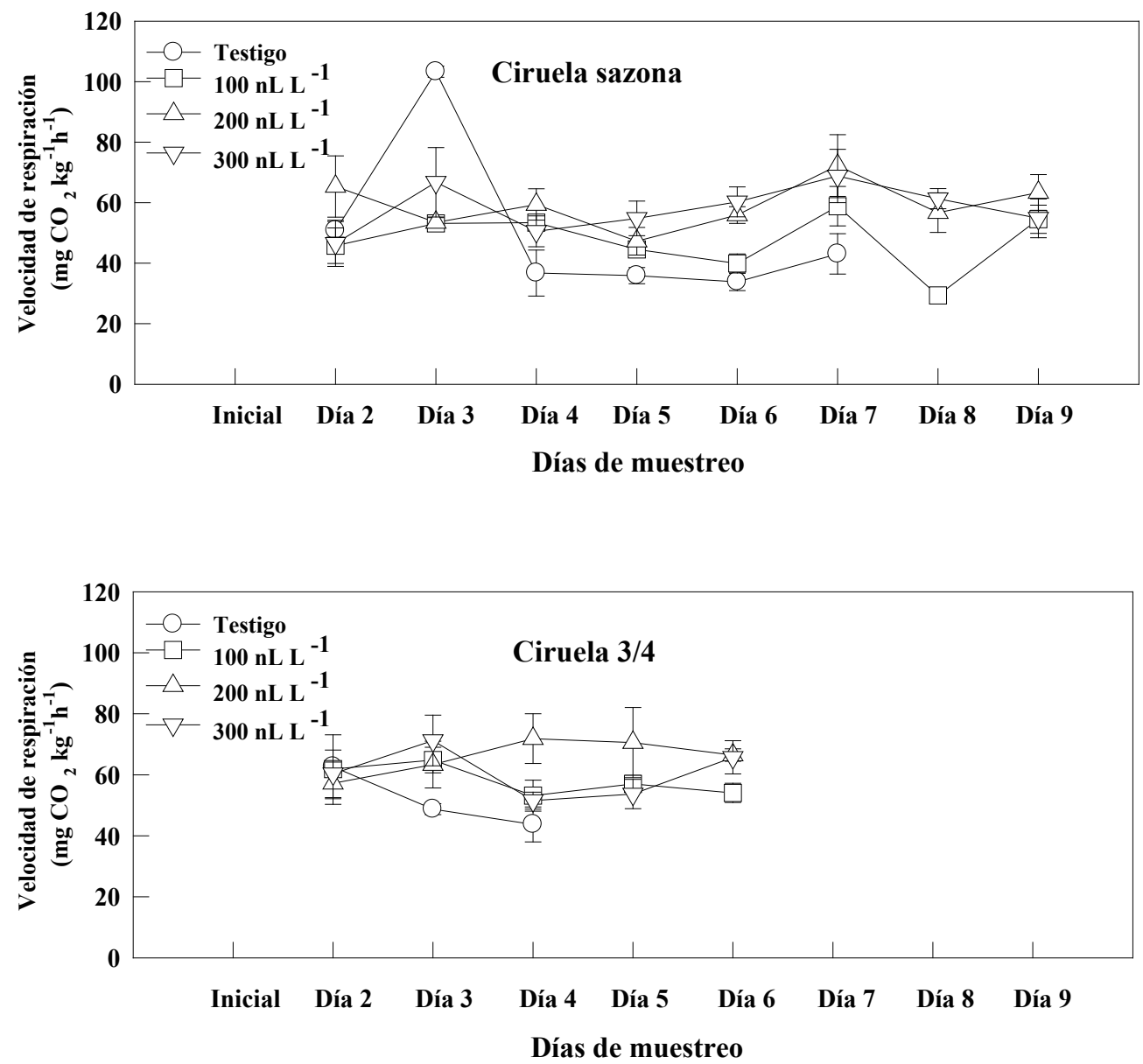

Figura 1. Velocidad de respiración en frutos de ciruela mexicana (Spondias purpurea L.) fenotipo amarillo, cosechados en dos estados de madurez (sazón y $3 / 4$ ) y almacenados a $22 \pm 2^{\circ} \mathrm{C}$ y $75 \pm 10 \% \mathrm{HR}$, en respuesta a la aplicación de 1-MCP. Cada punto representa la media \pm error estándar $(n=3)$. 
jaca y chicozapote o sapodilla se ha reportado que el 1-MCP en dosis de 40 a $300 \mathrm{~nL} \mathrm{~L}^{-1}$ disminuye la intensidad respiratoria y retrasa significativamente la aparición del pico climatérico (Quiping et al., 2006; Arévalo-Galarza et al., 2007; Mata-Montes de Oca et al., 2007). También Blankenship y Dole (2003) mencionaron que el 1-MCP reduce la velocidad de respiración o retrasa los incrementos en respiración en una gran variedad de especies. Otros investigadores han reportado que el 1-MCP retrasa significativamente el pico climatérico de respiración en ciruela de clima templado de las variedades 'Royal Zee,' 'Santa Rosa', 'Laetitia' y 'Harrow Sun' (Dong et al., 2002; Argenta et al., 2003; Manganaris et al., 2007).

En los frutos cosechados en tres cuartos de madurez, los del tratamiento testigo iniciaron con una tasa de $62.6 \mathrm{mg}$ de $\mathrm{CO}_{2} \mathrm{~kg}^{-1} \mathrm{~h}^{-1}$ que luego disminuyó hasta $43.7 \mathrm{mg}$ de $\mathrm{CO}_{2} \mathrm{~kg}^{-1}$ $\mathrm{h}^{-1}$ para el día cuatro de almacenamiento, en tanto que los frutos tratados con 1-MCP mantuvieron tasas entre 50 y 70 $\mathrm{mg}$ de $\mathrm{CO}_{2} \mathrm{~kg}^{-1} \mathrm{~h}^{-1}$ sin manifestar pico climatérico. Según Canto-Pereira et al. (2000), la ciruela amarilla (S. mombin L.) presenta un patrón climatérico de respiración para los estados uno y dos, mientras que los frutos cosechados en estado tres (madurados en el árbol) tienen una tasa inicial de $52 \mathrm{mg}$ de $\mathrm{CO}_{2} \mathrm{~kg}^{-1} \mathrm{~h}^{-1}$ que luego disminuye hasta $22 \mathrm{mg}$ de $\mathrm{CO}_{2} \mathrm{~kg}^{-1} \mathrm{~h}^{-1}$ en poco más de $24 \mathrm{~h}$ de almacenamiento, sin que se presentara el pico climatérico. Algo similar pudo haber sucedido en el presente estudio, ya que en los frutos cosechados en tres cuartos de madurez no se observó el pico climatérico en ninguno de los tratamientos.

\section{Pérdida de peso}

La pérdida de peso de la ciruela amarilla se redujo significativamente $(\mathrm{P} \leq 0.05)$ con la aplicación del $1-\mathrm{MCP}$ al fruto sazón, pero no indujo cambios en frutos de tres cuartos de maduración (Figura 2). En fruto sazón, hasta el día seis de almacenamiento no se detectaron diferencias de pérdida de peso entre frutos no tratados y los tratados con 1-MCP. No obstante, a partir del día siete los frutos tratados con $100 \mathrm{~nL}$ $\mathrm{L}^{-1}$ de 1-MCP mostraron un incremento significativo $(\mathrm{P} \leq$ $0.05)$ de la pérdida de peso (18.6\%) en comparación con las dosis de 200 y $300 \mathrm{~nL} \mathrm{~L}^{-1}$ (16.5 y $16.6 \%$, respectivamente). Esto indica que el 1-MCP es eficiente en dosis altas, respuesta atribuible a que las estas dosis disminuyen la tasa respiratoria.
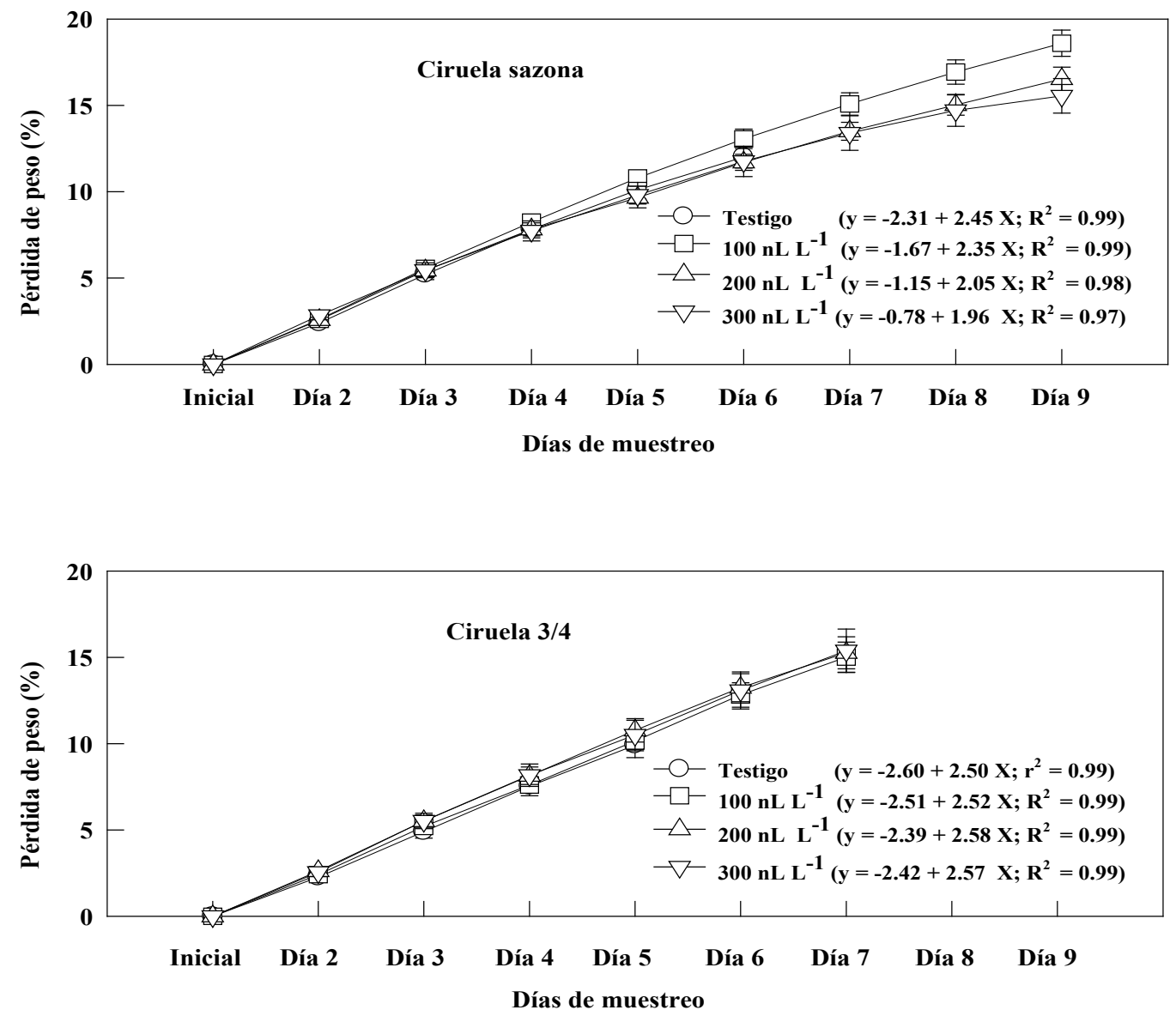

Figura 2. Pérdida de peso en frutos de ciruela mexicana (Spondias purpurea L.) fenotipo amarillo, cosechados en dos estados de madurez (sazón y $3 / 4$ ) y almacenados a $22 \pm 2{ }^{\circ} \mathrm{C}$ y $75 \pm 10 \% \mathrm{HR}$, en respuesta a la aplicación de 1-MCP. Cada punto representa la media \pm error estándar $(n=20)$. 
Resultados similares fueron obtenidos por Jeong et al. (2002) y Osuna-García et al. (2005a) en aguacate. Por el contrario, Arévalo-Galarza et al. (2007) en chicozapote y Mata-Montes de Oca et al. (2007) en jaca, reportaron que el 1-MCP no afectó la pérdida de peso de estos frutos. Por su parte Martínez-Romero et al. (2003) señalaron que en ciruela 'Santa Rosa' de tipo climatérico y en 'Golden Japan' de tipo no climatérico, el 1-MCP reduce la pérdida de peso en ambas variedades, efecto que dependió de la concentración en la variedad 'Santa Rosa' pero independiente de la concentración en 'Golden Japan'. Sin embargo, Salvador et al. (2003) reportaron que el 1-MCP no afecta la pérdida de peso en frutos de la ciruela 'Santa Rosa'. Con estos resultados, se puede inferir que para disminuir la pérdida de peso en frutos de ciruela mexicana amarilla la mejor dosis de 1-MCP es la de $200 \mathrm{~nL} \mathrm{~L}^{-1}$.

\section{Color externo}

El 1-MCP retrasó $(\mathrm{P} \leq 0.05)$ el desarrollo del color externo en ambos estados de madurez (Figura 3). En ciruela sazona tal efecto fue notorio a partir del día cinco de almacenamiento, cuando los frutos testigo mostraron un ángulo de matiz de 81.5 mientras que los tratados con 1-MCP mostraron valores de 91.1 a 99.9, que indican mantenimiento del color verde de cáscara. En la ciruela tres cuartos fue a partir del día cuatro cuando los frutos testigo mostraron valores de ángulo de matiz de 74.3, y los tratados tuvieron valores que oscilaron entre 84.8 y 88.2. En ambos estados de madurez a la cosecha, el 1-MCP demoró el desarrollo de color amarillo de los frutos hasta por $3 \mathrm{~d}$, lo que coincide con lo revelado por Blankenship y Dole (2003) quienes observaron que el 1-MCP previene o retrasa la degradación de clorofila y los cambios de color en una gran variedad de especies hortícolas y frutícolas. Jiang et al. (1999) en plátano (Musa paradisiaca L), Feng et al. (2000) en aguacate y Quiping et al. (2006) en sapodilla reportaron que el 1-MCP inhibe la degradación de clorofila y retrasa el cambio de color de cáscara, lo cual atribuyeron a inhibición de los efectos inducidos por el etileno. En ciruela de los tipos Europea y Japonesa de las variedades 'Royal Zee', 'Santa Rosa', 'Golden Japan, 'Laetitia', 'President', 'Fortune' y 'Angeleno', varios investigadores reportaron que el 1-MCP aplicado en dosis de 250 a $1000 \mathrm{~nL} \mathrm{~L}^{-1}$ retrasa el desarrollo de color de cáscara y pulpa (Dong et al., 2002; Argenta et al., 2003; Martínez-Romero et al., 2003; Salvador et al., 2003; Menniti et al., 2004; Valero et al., 2004).
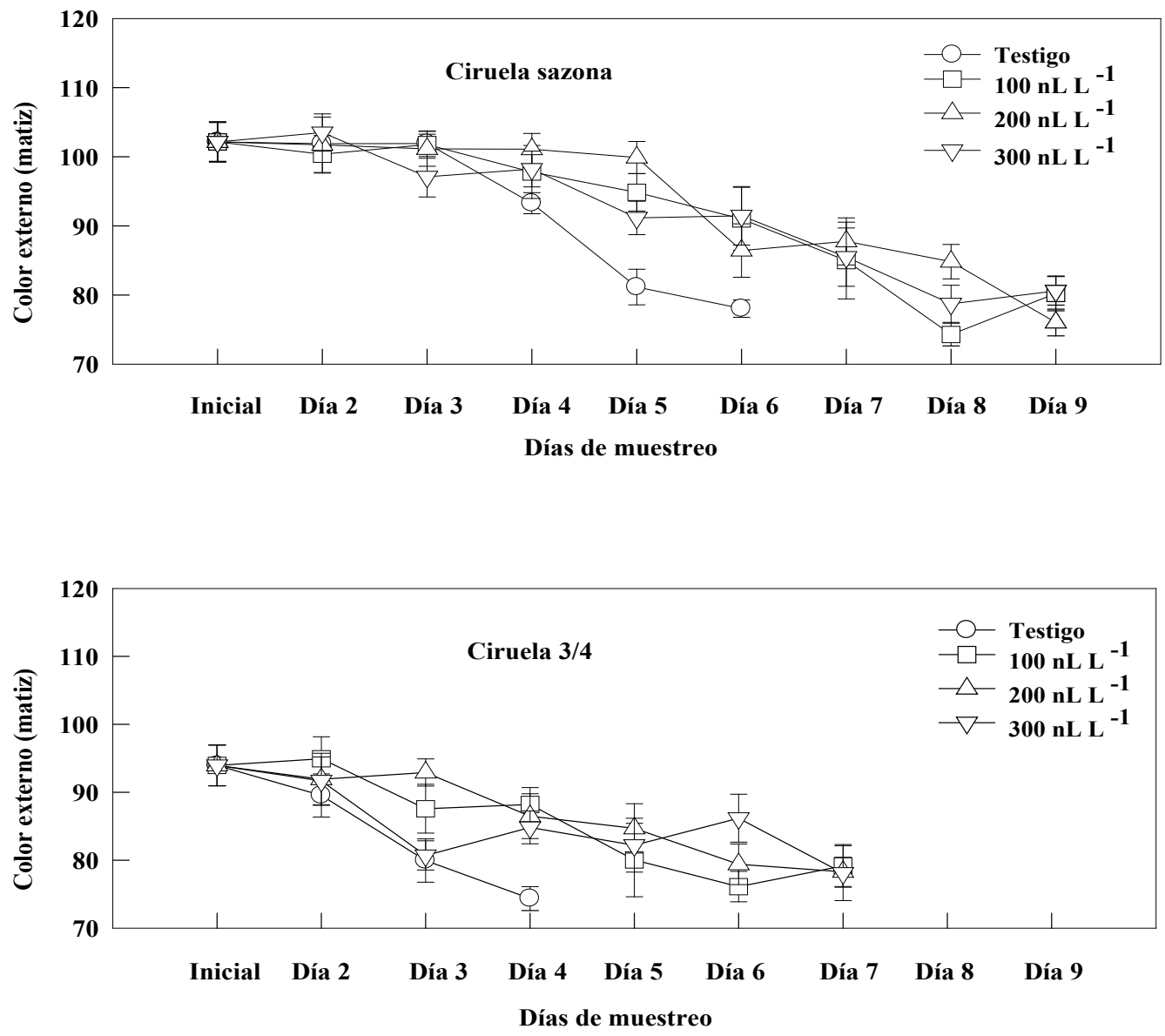

Figura 3. Color externo (matiz) en frutos de ciruela mexicana (Spondias purpurea L.) fenotipo amarillo, cosechados en dos estados de madurez (sazón y $3 / 4$ ) y almacenados a $22 \pm 2^{\circ} \mathrm{C}$ y $75 \pm 10 \% \mathrm{HR}$, en respuesta a la aplicación de 1-MCP. Cada punto representa la media \pm error estándar $(\mathbf{n}=6)$. 


\section{Firmeza}

El 1-MCP retrasó también $(\mathrm{P} \leq 0.05)$ el ablandamiento de los frutos (Figura 4). En frutos cosechados en estado de madurez sazón, al día seis los del tratamiento testigo mostraban una firmeza de sólo $28.7 \mathrm{~N}$, inferior a la de los tratados que en ese momento tenían una firmeza entre 41.1 y 57.5 N. Los frutos testigo llegaron a madurez de consumo (entre 20 y $30 \mathrm{~N}$ ) al día seis, mientras que los tratados con 1-MCP en cualquiera de sus dosis lo hicieron hasta el día nueve. Un resultado similar se observó en los frutos cosechados en el estado de madurez de tres cuartos, ya que los frutos testigo llegaron a madurez de consumo en $4 \mathrm{~d}$ en tanto que los tratados con 1-MCP lo hicieron hasta el día siete. En ambos estados de madurez el 1-MCP mantuvo hasta por 3 $\mathrm{d}$ adicionales la firmeza de frutos, lo que les confiere mayor resistencia al transporte y la posibilidad de llegar a mercados más distantes con menores riesgos de pérdidas postcosecha, y supera con mucho los $4 \mathrm{~d}$ de vida útil de frutos de
Spondia cosechados en diversos grados de madurez (DíazPérez et al., 1999; Pérez-López et al., 2004).

Resultados similares fueron encontrados por Mata-Montes de Oca et al. (2007) en jaca, ya que mencionan que con dosis de 100 y $300 \mathrm{~nL} \mathrm{~L}^{-1}$ el 1-MCP prolongó por 8 y $12 \mathrm{~d}$ más la firmeza, tanto en la pulpa como en el fruto entero. También Quiping et al. (2006) encontraron que el 1-MCP retrasa el ablandamiento de sapodilla, ya que al día 12 los frutos tratados mostraban una firmeza de $37.8 \mathrm{~N}$ en tanto que los del testigo sólo alcanzaban $8.5 \mathrm{~N}$. El retraso del ablandamiento se atribuye a la inhibición de las enzimas involucradas en la degradación de la pared celular, como: celulosa y pectin metilesterasa, poligalacturonasa, 1 , 4- $\beta$-Dglucanasa y $\beta$-galactosidasa así como exopoligalacturonasa, endopoligalacturonasa y pectin esterasa (Feng et al., 2000; Dong et al., 2001; Jeong et al., 2002; Manganaris et al., 2007; Khan y Singh, 2008).
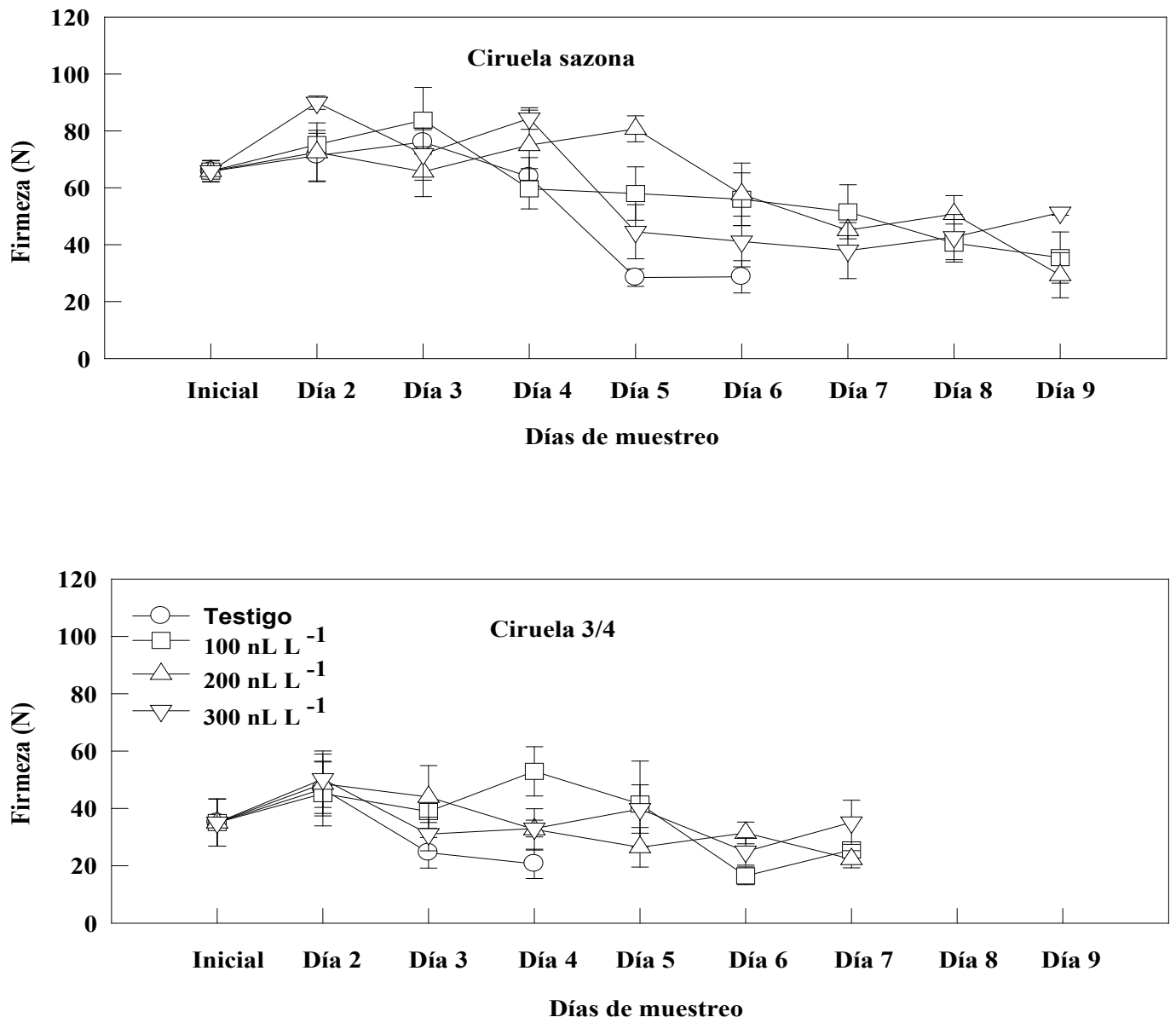

Figura 4. Firmeza en frutos de ciruela mexicana (Spondias purpurea L.) fenotipo amarillo, cosechados en dos estados de madurez (sazón y ${ }^{3 / 4}$ ) y almacenados a $22 \pm 2^{\circ} \mathrm{C}$ y $75 \pm 10 \% \mathrm{HR}$, en respuesta a la aplicación de 1-MCP. Cada punto representa la media \pm error estándar $(n=6)$. 


\section{Sólidos solubles totales (SST)}

El 1-MCP no afectó el desarrollo de los SST (Figura 5), ya que en ambos estados de corte los frutos tratados llegaron a madurez de consumo con valores similares al testigo. En frutos sazones los testigos tenían un valor de $14.1^{\circ} \mathrm{Bx}$ al día seis de almacenamiento, en tanto que los tratados oscilaron de 13.7 a $14.4^{\circ} \mathrm{Bx}$ al día nueve de almacenamiento. En los frutos cosechados en madurez de tres cuartos, los testigos llegaron a $12.5^{\circ} \mathrm{Bx}$ al día cuatro de almacenamiento mientras que los tratados alcanzaron de 13.6 a $13.8^{\circ} \mathrm{Bx}$ al día siete de almacenamiento.

Se han reportado resultados contrastantes en cuanto al efecto del 1-MCP sobre los SST. Según Hofman et al. (2001) y Quiping et al. (2006), en papaya (Carica papaya L.) y sapodilla el 1-MCP incrementó los valores, mientras que otros autores han reportado que en papaya, mango y jaca el 1-MCP sólo retrasa la evolución de los SST (Osuna-García et al., 2005b; Osuna-García et al., 2005c; Mata-Montes de Oca et al., 2007). En ciruela diversos autores han reportado que el 1-MCP tiene poco o ningún efecto en el contenido de SST en diversas variedades del tipo Europea y Japonesa (Argenta et al., 2003; Salvador et al., 2003; Menniti et al., 2004; Manganaris et al., 2007; Khan y Singh, 2008).

\section{CONCLUSIONES}

El 1-MCP en cualquiera de sus dosis (100, 200 ó $300 \mathrm{~nL}$ $\mathrm{L}^{-1}$ ) alarga la vida de anaquel de los frutos de ciruela mexicana del fenotipo amarillo hasta por $3 \mathrm{~d}$ adicionales con respecto al testigo no tratado. Ello permite alargar la vida de anaquel a $9 \mathrm{~d}$ en frutos sazones y a $7 \mathrm{~d}$ en frutos de tres cuartos. El 1-MCP disminuye la respiración en todos los casos y reduce la pérdida de peso en frutos sazones, pero no en frutos en tres cuartos de maduración; además, el 1-MPC retrasa el desarrollo del color externo y permite mantener la firmeza del fruto sin afectar el contenido de sólidos solubles totales.

\section{BIBLIOGRAFÍA}

AOAC, Association of Official Agricultural Chemists (1984) Official Methods of Analysis. 14th ed. Published for the Association of Official Analytical Chemist Inc. Arlington, VA. 22209 USA. 1006 p.

Arévalo-Galarza L, B Bautista-Reyes, C Saucedo-Veloz, T MartínezDamián (2007) Almacenamiento refrigerado y aplicaciones de 1-Metilciclopropeno (1-MCP) en frutos de chicozapote (Manilkara sapota (L.) P. Royen). Agrociencia 41:469-477.

Argenta L C, J G Krammes, C A Megguer, C V T Amarante, J Mattheis (2003) Ripening and quality of 'Laetitia' plums following harvest and cold storage as affected by inhibition of ethylene action. Pesq.
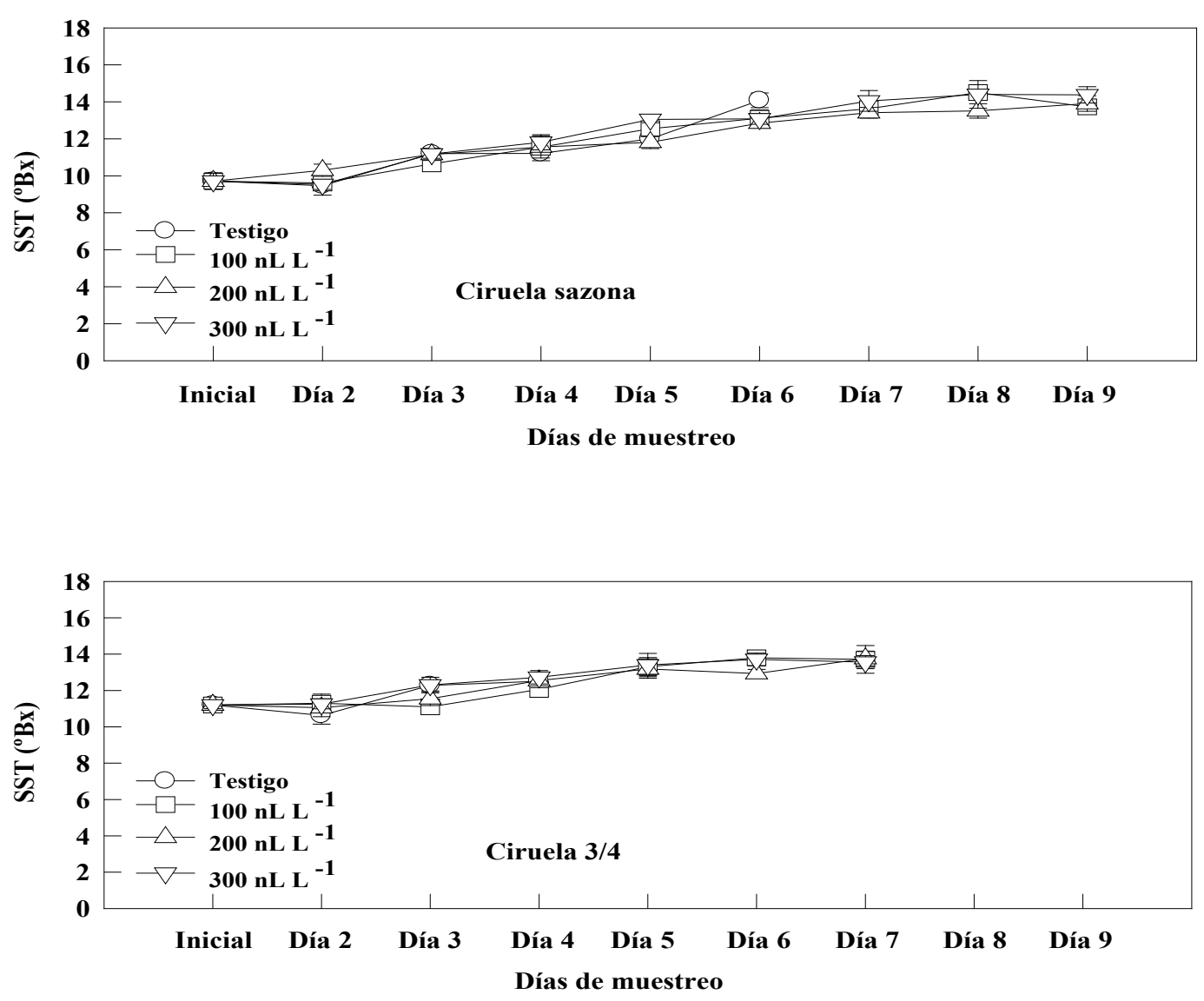

Figura 5. Contenido de sólidos solubles en frutos de ciruela mexicana (Spondias purpurea L.) fenotipo amarillo, cosechados en dos estados de madurez (sazón y $3 / 4$ ) y almacenados a $22 \pm 2{ }^{\circ} \mathrm{C}$ y $75 \pm 10 \% \mathrm{HR}$, en respuesta a la aplicación de 1-MCP. Cada punto representa la media \pm error estándar $(n=6)$. 
Agrop. Bras. 38:1139-1148.

Blankenship S, J Dole (2003) 1-Methylcyclopropene: a review. Postharv. Biol. Technol. 28:1-25.

Canto-Pereira M E, H A Cunha-Filgueiras, R Elesbao-Alves (2000) Actividad respiratoria y producción de etileno postcosecha de ciruela mexicana y jobo. Rev. Iberoam. Tecnol. Post. 2:155-160.

Díaz-Pérez J C, R Zavaleta, S Bautista, B Aguilar, V Sebastián (1999) Cambios físico-químicos de ciruela mexicana (Spondias purpurea L.) cosechada en dos diferentes estados de madurez. Rev. Iberoam. Tecnol. Post. 2:19-24

Dong L, Z Hong-Wei, L Sonego, A Lers, S Lurie (2001) Ripening of 'Red Rosa' plums: effect of ethylene and 1-methylcyclopropene. Aust. J.Plant Physiol. 28:1039-1045.

Dong L, S Lurie, Z Hong-Wei (2002) Effect of 1-methylcyclopropene on Ripening of 'Canino' apricots and 'Royal Zee' plums. Postharv. Biol. Technol. 24:135-145.

Dowdy S, S Wearden (1991) Statistics for Research. John Wiley \& Sons. New York, USA. 629 p.

Environmental Protection Agency (2002) Federal Register, July 26. Environ. Protect. Agency 67(144):48796-48800.

Feng X, A Apelbaum, E Sisler, R Goren (2000) Control of ethylene responses in avocado fruits with 1-methylcyclopropene. Postharv. Biol. Technol. 20:143-150.

Hofman P J, M Jobin-Décor, G F Meiburg, A J Macnish, D C Joyce (2001) Ripening and quality responses of avocado, custard apple, mango and papaya fruit to 1-methylcyclopropene. Aust. J. Exp. Agric. 41:567-572.

Jeong J, D J Huber, S A Sargent (2002) Influence of 1-methylcyclopropene (1-MCP) on ripening and cell-wall matrix polysaccharides of avocado (Persea americana) fruit. Postharv. Biol. Technol. 25:241256.

Jiang Y, D Joyce, A Macnish (1999) Extension of the shelf-life of banana fruit by 1-Methylcyclopropene in combination with polyethylene bags. Postharv. Biol. Technol. 16:187-193.

Jiang Y, D Joyce (2000) Effects of 1-methylcyclopropene alone and in combination with polyethylene bags on the postharvest life of mango fruits. Ann. Appl. Biol. 137:321-327.

Khan A S, Z Singh (2008) 1-Methylcyclopropene application and modified atmosphere packaging affect ethylene biosynthesis, fruit softening, and quality of 'Tegan Blue' Japanese plum during cold storage. J. Amer. Soc. Hort. Sci. 133:290-299.

Kluge R A, A P Jacomino, R M Ojeda, A Brackmann (2002) Avocado ripening inhibition by 1-methylcyclopropene. Pesq. Agrop. Bras. 37:895-901.

Manganaris G A, A R Vicente, C H Crisosto, J M Labavitch (2007) Effect of dips in a Methylcyclopropene-generating solution on 'Harrow Sun' plums stored under different temperature regimes. J. Agric. Food Chem. 55:7015-7020.

Martínez-Romero D, E Dupille, F Guillén, J M Valverde, M Serrano, D Valero (2003) 1-Methylcyclopropene increases storability and shelf life in climacteric and nonclimacteric plums. J. Agric. Food Chem. 51:4680-4686.

Mata-Montes de Oca M, J A Osuna-García, A Hernández-Estrada, M Ochoa-Villarreal, B Tovar-Gómez (2007) Efecto del 1-Metilciclopropeno (1-MCP) sobre la fisiología y calidad de frutos de Jaca (Artocarpus heterophyllus Lam). Rev. Chapingo S. Hort. 13:165170.

Menniti AM, R Gregori, I Donati (2004) 1-Methylcyclopropene retards postharvest softening of plums. Postharv. Biol. Technol. 31:269275.

Montalvo-González E, H S García, M Mata-Montes de Oca, B TovarGómez (2011) Efecto de la luz en ciruela mexicana manejada en diferentes condiciones de almacenamiento. CyTA J. Food 9:65-70.

Osuna-García J A, J A Beltrán, V Vázquez-Valdivia (2005a) Efecto del 1-Metilciclopropeno (1-MCP) sobre el comportamiento postcosecha del aguacate 'Hass'. Rev. Fitotec. Mex. 28:1-8.

Osuna-García J A, J A Beltrán, M A Urías-López (2005b) Efecto del 1-Metilciclopropeno (1-MCP) sobre la vida de anaquel y calidad de mango para exportación. Rev. Fitotec. Mex. 28:271-278.

Osuna-García J A, J A Beltrán, M H Pérez-Barraza (2005c) Mejoramiento de la vida de anaquel y calidad de papaya 'Maradol' con el 1-Metilciclopropeno (1-MCP). Rev. Chapingo S. Hort. 11:7-12.

Pérez-López A, C Saucedo-Veloz, M L Arévalo-Galarza, A MuratallaLúa (2004) Efecto del grado de madurez en la calidad y vida postcosecha de ciruela mexicana (Spondias purpurea L.). Rev. Fitotec. Mex. 27:133-139.

Quiping Z, X Wenshui, Y Jiang (2006) Effects of 1-Methylcyclopropene treatments on ripening and quality of harvested sapodilla fruit. Food Technol. Biotechnol. 44:535-539.

Rebolledo-Robles H H (2002) Manual SAS por Computadora: Análisis Estadísticos de Datos Experimentales. Ed. Trillas, México. 208 p.

Rohm and Haas (2002) SmartFresh ${ }^{\mathrm{TM}}$ tabs. Material Safety Data Sheet. 7 p.

Salvador A, J Cuquerella, J M Martinez-Jávega (2003) 1-MCP treatment prolongs postharvest life of 'Santa Rosa' plums. J. Food Sci. 68:1504-1510.

SAGARPA, Secretaría de Agricultura, Ganadería, Desarrollo Rural, Pesca y Alimentación (2008) Centro de Estadística Agropecuaria. Delegación Estatal. Tepic, Nayarit.

SIAP, Subsistema de Información Agrícola-SAGARPA (2007) Disponible en: http://www.siap.sagarpa.gob.mx. (Diciembre de 2007).

Sisler E C, S M Blankenship (1996) Method of Counteracting an Ethylene Response in Plants. U. S. Patent 5518988

Sisler E C, M Serek (1997) Inhibitors of ethylene responses in plants at the receptor level: Recent developments. Physiol. Plant. 100:577-582.

Sisler E C, M Serek (1999) Compounds controlling the ethylene receptor. Bot. Bull. Acad. Sin. 40:1-7.

Valero D, D Martínez-Romero, J M Valverde, F Guillén, S Castillo, M Serrano (2004) Could the 1-MCP treatment effectiveness in plum be affected by packaging? Postharv. Biol. Technol. 34:295-303. 